\title{
La priorale Saint-Pierre de Souvigny (Allier) : étude archéologique de la nef, seconde tranche
}

Pascale Chevalier, Sébastien Bully, Morana Čaušević-Bully, Mathias Dupuis, Laurent Fiocchi et Audrey Baradat

\section{OpenEdition}

1 Journals

Édition électronique

URL : https://journals.openedition.org/cem/6382

DOI : $10.4000 /$ cem. 6382

ISSN : 1954-3093

Éditeur

Centre d'études médiévales Saint-Germain d'Auxerre

Édition imprimée

Date de publication : 15 août 2008

ISSN : 1623-5770

Référence électronique

Pascale Chevalier, Sébastien Bully, Morana Čaušević-Bully, Mathias Dupuis, Laurent Fiocchi et Audrey Baradat, «La priorale Saint-Pierre de Souvigny (Allier) : étude archéologique de la nef, seconde

tranche ", Bulletin du centre d'études médiévales d'Auxerre / BUCEMA [En ligne], 12 | 2008, mis en ligne le 15 juillet 2008, consulté le 22 septembre 2022. URL : http://journals.openedition.org/cem/6382 ; DOI : https://doi.org/10.4000/cem.6382

Ce document a été généré automatiquement le 22 septembre 2022.

\section{(c)}

Creative Commons - Attribution - Pas d'Utilisation Commerciale - Partage dans les Mêmes Conditions 4.0 International - CC BY-NC-SA 4.0

https://creativecommons.org/licenses/by-nc-sa/4.0/ 


\title{
La priorale Saint-Pierre de Souvigny (Allier) : étude archéologique de la nef, seconde tranche
}

\author{
Pascale Chevalier, Sébastien Bully, Morana Čaušević-Bully, Mathias \\ Dupuis, Laurent Fiocchi et Audrey Baradat
}

1 Comme en 2006, l'opération archéologique a été menée dans le cadre de la réfection intérieure des cinq vaisseaux de la nef de la priorale clunisienne par la CRMH d'Auvergne ${ }^{1}$. L'intervention de quatre mois, toujours motivée par les risques de destruction générés par les travaux, avait pour objectif minimal de dégager, sur 30 à $35 \mathrm{~cm}$ de profondeur, le vaisseau central et les deux collatéraux sud de la nef; soumise au calendrier du chantier, elle a débuté en avril, s'est poursuivie en juin et juillet, pour s'achever fin septembre ${ }^{2}$. On se reportera à la notice de 2006 pour une brève description du cadre architectural de l'intervention ${ }^{3}$.

Un calendrier très resserré et des problèmes de circulation ont imposé des choix stratégiques. Nous avons limité la fouille du vaisseau central entre la première paire de supports et la travée du tombeau des saints abbés à un décapage mécanique sur $25 / 30 \mathrm{~cm}$ de profondeur, poursuivi à la main sur les $5 / 10 \mathrm{~cm}$ suivants; nous avons abandonné la fouille du bas-côté interne sud, de la première et quatrième travée du collatéral externe, ainsi que celle de la travée d'entrée et de la deuxième travée du vaisseau central, en laissant à l'entreprise le soin des terrassements. Ceci nous a permis de procéder à trois sondages profonds à des emplacements limités, justifiés par des problématiques scientifiques. Le premier de ces sondages était une tranchée traversant le vaisseau médian au niveau de la première paire de support, le deuxième couvrait la $2^{\mathrm{e}}$ et la $3^{\mathrm{e}}$ travée du collatéral externe sud, le troisième accompagnait les compléments de fouille autour du tombeau des saints abbés dans la dernière travée du vaisseau central, du collatéral interne et la moitié du bas-côté externe (fig. 1). À cet endroit, les trois piles romanes limitant le grand transept ont été reprises en sous-œuvre au béton armé dans les années 1930-1950; il en résulte deux profondes fosses de 5 et $10 \mathrm{~m}$ de largeur nord-sud, séparées par un lambeau d'environ $1 \mathrm{~m}$ de stratigraphie en place, 
juste dans l'axe longitudinal de l'édifice. En vidant leur comblement, nous avons obtenu, sur deux de leurs parois, des coupes stratigraphiques d'un intérêt majeur, sur une puissance maximale de $1,70 \mathrm{~m}$.

Nous exposerons succinctement les nombreux résultats obtenus en 2007 en partant des périodes les plus anciennes.

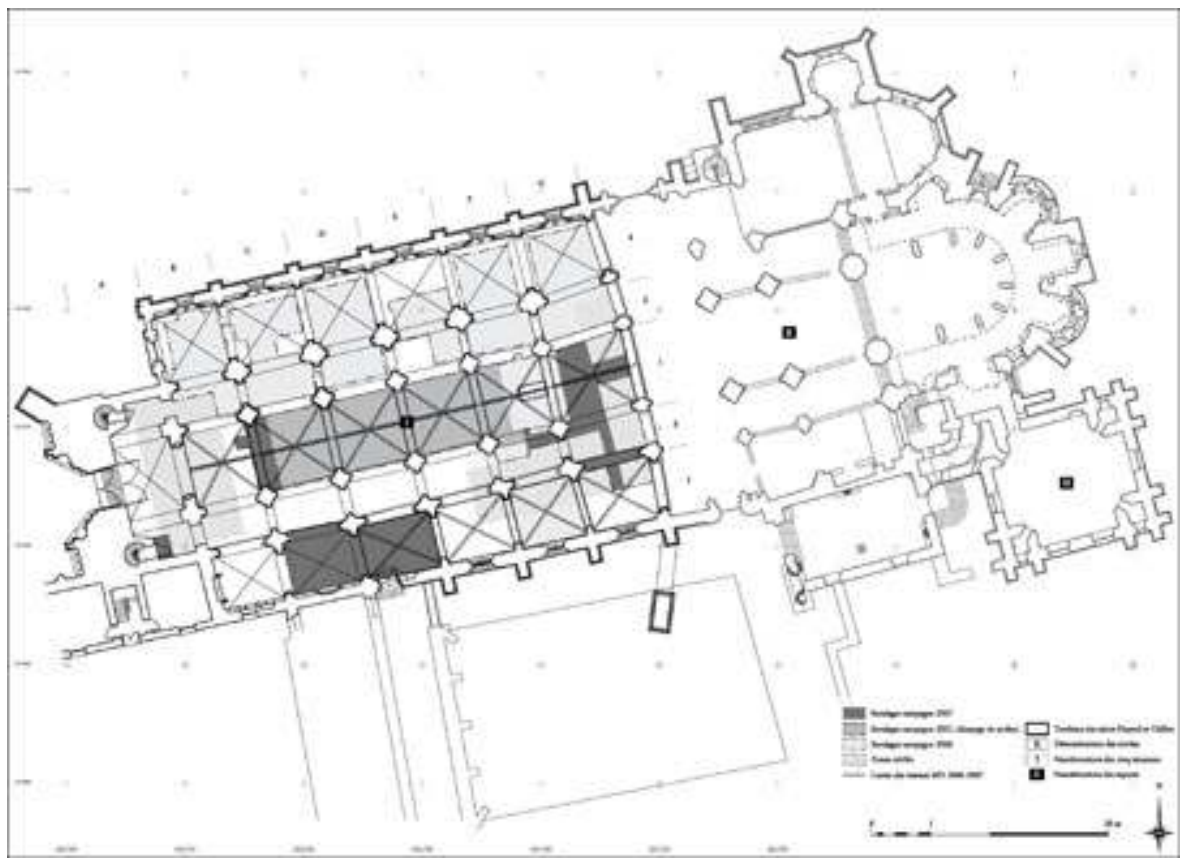

Fig. 1 - Plan de la priorale Saint-Pierre de Souvigny avec l'implantation des différentes interventions de 2007 (del. L. Fiocchi).

Une église de la seconde moitié du $\mathrm{X}^{\mathrm{e}}$ siècle

4 Tout d'abord des vestiges importants et assez inattendus d'une phase de l'église antérieure à l'an Mil (nef de l'abbé Mayeul, 954-994) ont été mis au jour : plusieurs segments du gouttereau sud en petit appareil, plus ou moins régulier, doté à son extrémité orientale d'une porte - menant au cloître et aux bâtiments monastiques -, dont le montant ouest, au moins, est bordé par un pilastre et devant laquelle se trouve une sépulture «de seuil» en sarcophage; les fondations de la portion centrale d'un mur transversal - au niveau de la future première paire de piles romanes - percé d'une très large ouverture, qui constitue la façade originelle ou un mur de refend limitant à l'est une possible avant-nef primitive. Nous rapprochons ces maçonneries de deux murs découverts en 2006 : l'extrémité orientale du gouttereau nord et la suite vers le nord du mur de "façade", ce qui nous donne une nef unique charpentée très légèrement trapézoïdale, mesurant 31,90 x 15,20/15,80 m hors œuvre. Le gouttereau sud est lié à un radier horizontal, qui paraît s'étendre sur la surface intérieure du bâtiment et fonctionner avec un mur arasé, orienté est-ouest, observé en coupe sept mètres vers le nord, dans une des fosses du $\mathrm{XX}^{\mathrm{e}}$ siècle. À proximité de cette dernière maçonnerie, on a dégagé une courte portion d'un mur perpendiculaire au précédent, bordé à l'ouest par une sépulture d'enfant orientée nord-sud, et le blocage d'un mur oblique, toujours dans la paroi de la fosse sud de reprise du $\mathrm{XX}^{\mathrm{e}}$ siècle. On associera à cette campagne de construction cinq fours à chaux circulaires, implantés en enfilade grossièrement nordouest sud-est, dont trois ont été identifiés juste au-delà la ligne de la "façade " occidentale, dans le collatéral externe sud, le vaisseau central et, en 2006, dans le bascôté interne nord où il a été pris à tort pour un plancher carbonisé (datation $\mathrm{C}^{14}$ 
910-950) ${ }^{4}$. Le four méridional prend appui sur les fondations d'un mur en petit appareil parallèle au gouttereau primitif et situé à $5 \mathrm{~m}$ au sud de ce dernier. Ce mur appartenant aux bâtiments monastiques du $\mathrm{X}^{\mathrm{e}}$ siècle est conservé en élévation sur près de $2 \mathrm{~m}$ car il a été repris au tout début du XII ${ }^{\mathrm{e}}$ siècle comme gouttereau sud de la $1^{\text {re }}$ et de la $2^{\mathrm{e}}$ travée du nouveau collatéral ; son tracé peut avoir déterminé la largeur des bas-côtés externes et il indique que le quadrilatère du cloître - s'il existe déjà - s'étend alors à une certaine distance du flanc sud de la nef.

La priorale au $\mathrm{XI}^{\mathrm{e}}$ siècle : architecture et aménagements liturgiques et funéraires

5 Les travaux entamés par l'abbé Odilon (994-1049) conduisent à la reconstruction de la nef: on remploie une bonne partie du gouttereau sud et on s'appuie sur le mur nord arasé afin de lui rendre une forme rectangulaire classique; la façade est avancée de $13,70 \mathrm{~m}$ vers l'ouest afin de créer une avant-nef - ou de reprendre celle que nous conjecturions plus haut? La nef est rapidement soumise à un violent incendie dont témoigne la couche de charbon recouvrant le premier sol que nous avons observé dans tout le vaisseau central ; à la datation $\mathrm{C}^{14}$, calibrée 890-1000, ne prenant pas en compte le travail du bois de charpente, on rajoutera une bonne quarantaine d'années. L'église semble avoir été aussitôt restaurée avec la construction d'un mur mince, parallèle au gouttereau sud presque dans l'alignement de la future colonnade, dont la fonction n'est pas déterminée. Cette nef charpentée rénovée accueille, peut-être en 1063, le sarcophage de saint Odilon et le premier monument funéraire ${ }^{5}$, avant sa tripartition dans le troisième quart du $\mathrm{XI}^{\mathrm{e}}$ siècle.

6 Nous avons pu affiner en 2007 les observations effectuées en 2002 autour du tombeau des saints abbés et ainsi mieux situer en chronologie relative les évolutions complexes ayant affecté le premier monument funéraire. La barrière trapézoïdale, reposant sur un stylobate de grès qui encadrait le sarcophage de remploi, sans doute primitivement exposé à la hauteur du sol, est remaniée au moment de l'implantation des deux files de piles composites, créant trois vaisseaux dans la nef. On arase alors la moitié ouest des longs côtés de la barrière afin de ménager deux accès, au moins visuels, vers le sarcophage. Le stylobate massif de la façade ouest du chœur liturgique vient s'appuyer à l'est du monument funéraire primitif, entre les piles qui séparent la $6^{\mathrm{e}}$ et la $7^{\mathrm{e}}$ travée du vaisseau médian. Deux portillons sont ménagés entre les piles et les angles du monument, où, quelques années plus tard, on installe les quatre poteaux d'un ciborium en bois. La $6^{\mathrm{e}}$ travée du collatéral paroissial nord est isolée de la zone autour du tombeau par un muret. Un autel, au titulaire inconnu, est ensuite édifié cinq mètres à l'est dans le chœur liturgique, à la limite de la croisée du transept dans l'axe de la nef ; son socle rectangulaire maçonné est longé en façade par trois colonnettes. Le baldaquin $\mathrm{du}$ tombeau du saint abbé brûle sans doute dans le dernier quart du XI ${ }^{e}$ siècle. Cet incendie, assez localisé mais également perceptible vers l'est dans la dernière travée de la nef, impose une restauration du monument funéraire et du muret de partition vers le vaisseau paroissial, ainsi que l'abandon de la première barrière de chancel. C'est trois mètres plus à l'est qu'est aménagée la nouvelle façade du chœur, cette fois maçonnée et soigneusement enduite sur son parement ouest. Curieusement, elle ne présente pas d'accès visible, l'axe longitudinal du vaisseau étant même marqué par la présence, au revers du socle conservé, du négatif d'un emmarchement en bois. En 1095, après ces travaux, Urbain II transfère le corps de saint Mayeul dans le tombeau rénové. La priorale au XII ${ }^{\mathrm{e}}$ siècle : architecture et aménagements liturgiques devant le tombeau des saints abbés 
7 Au tournant du XI ${ }^{\mathrm{e}}$ et du XII ${ }^{\mathrm{e}}$ siècle, la nef est agrandie d'abord au nord puis au sud d'un bas-côté externe - comme au nord, on perce dans le gouttereau méridional du $\mathrm{XI}^{\mathrm{e}}$ siècle une série de six grandes arcades. Six piles quadrilobées, plus régulières et mieux pensées qu'au nord, sont créées à partir de tronçons du gouttereau, chacun comprenant, du côté nord, une colonne engagée et son chaînage et, à l'opposé, le contrefort extérieur. On flanque chaque tronçon d'une demi-colonne adossée sur dosseret et le contrefort est inclus au sud dans un large pilastre doté d'une $4^{\mathrm{e}}$ colonne engagée. Le nouveau gouttereau sud récupère sur deux travées le mur du $\mathrm{X}^{\mathrm{e}}$ siècle cité supra et comporte, au niveau de la $3^{\mathrm{e}}$ travée, un exutoire voûté pour un drain, qui évacue vers le préau du cloître les eaux ruisselant en sous-sol. Au-devant de l'exutoire est aménagé un dallage rectangulaire de $2 \mathrm{~m}^{2}$, qui demeure le seul vestige de ce drain ${ }^{6}$. Ces travaux s'achèvent par un terrassement et un rehaussement général du sol. Dans la dernière travée orientale, on crée alors un nouveau dispositif délimitant le chœur liturgique. On isole le vaisseau médian des collatéraux septentrionaux par une barrière épaisse de $28 \mathrm{~cm}$, faisant retour vers le sud pour constituer la nouvelle façade occidentale, là encore de forme assez inattendue, dont nous n'avons documenté que des négatifs et des fosses d'arrachement. Cette façade reprend l'alignement de celle du deuxième chancel, mais comporte un étroit accès central $(60 \mathrm{~cm})$ bordé par deux massifs d'autel quadrangulaires (dim. rest. 1,60 x $1 \mathrm{~m}$; fig. 2). Vers le sud, après un court segment de barrière mince, prend place un massif maçonné mesurant $1,38 \mathrm{~m}$ de largeur, dont la fonction n'est pas élucidée (fig. 2), puis, au-delà d'un accès ouvrant sur le collatéral interne sud, la barrière (ép. $28 \mathrm{~cm}$ ) fait un léger décrochement vers l'est et s'étend encore sur 2,50 $\mathrm{m}$ de longueur dans le bas-côté externe - probablement pour protéger un nouvel accès des religieux vers le cloître. De même que la précédente et que le monument funéraire, cette troisième barrière comporte un décor d'enduit peint imitant des placages de marbres colorés ${ }^{7}$. Nous avons documenté en 2006 l'autel SaintNicolas à l'extrémité est du bas-côté externe nord, dévolu aux fonctions paroissiales. Son implantation quelque temps après la pentapartition de la nef conduit à obturer par une maçonnerie soignée, épaisse de $40 \mathrm{~cm}$, l'espace compris entre l'angle nord-ouest du chœur liturgique et la pile située à l'ouest.

On opère une refonte complète du tombeau et des aménagements liturgiques situés juste à l'est à la fin du chantier de reconstruction roman, dans les années 1170. Outre le remplacement du premier monument par un caveau surmonté par un sépulcre monumental, dont il ne nous reste rien, on ramène la façade du chœur liturgique au niveau de la première barrière de chancel, entre les deux piles séparant la $6^{\mathrm{e}}$ et la $7^{\mathrm{e}}$ travée de la nef. Cette quatrième barrière est impressionnante avec 2,50/2,70 $\mathrm{m}$ de hauteur et un couronnement sculpté de personnages en façade et d'arcades sur les retours latéraux ${ }^{8}$. Deux accès permettent aux moines de circuler autour de la tombe sainte. À peine $1 \mathrm{~m}$ à l'est, on aménage dans le chœur, à la même date, une plate-forme mesurant $4 \times 4 \mathrm{~m}$, légèrement convexe à l'ouest, dont les côtés reçoivent des stalles et sur laquelle on implante, à l'aplomb de son emplacement précédent, mais après un hiatus important, le socle maçonné d'un nouvel autel secondaire axial (1. est-ouest $1,04 \mathrm{~m}$ ). La plate-forme est abandonnée, arasée et couverte d'une couche de glaise indurée au XIII ${ }^{\mathrm{e}}$ siècle, lors de l'ultime réfection du tombeau des saints abbés, qui est doté de gisants et encadré de deux autels qui ferment les entrées latérales de la façade occidentale du chœur ; l'autel d'axe reste en usage jusqu'à une date indéterminée - les sols qui auraient pu nous renseigner ont été nivelés au milieu du XIX ${ }^{\mathrm{e}}$ siècle. 


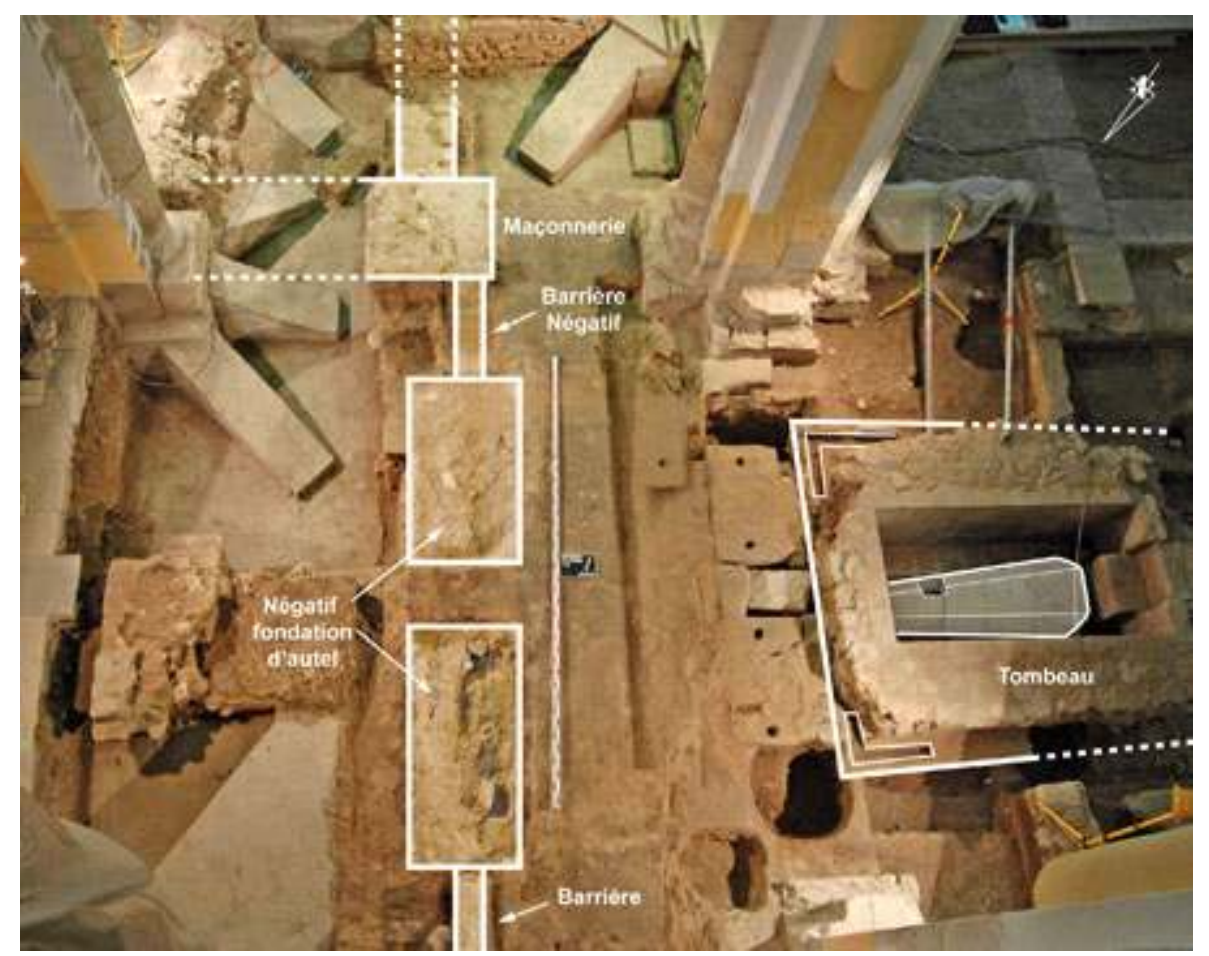

Fig. 2 - Vue plongeante sur la dernière travée de la nef : les vestiges de la troisième barrière de chœur (cliché L. Fiocchi, DAO D. Vuillermoz).

Les chapelles funéraires gothiques dans le collatéral externe sud

Le sondage mené dans la $2^{\mathrm{e}}$ et $3^{\mathrm{e}}$ travée du bas-côté externe sud avait pour objectif de documenter deux chapelles funéraires gothiques installées à la fin du XIV siècle et, pour les phases antérieures à la construction du collatéral du début du XII ${ }^{e}$ siècle, de retrouver la galerie nord du cloître de l'église d'odilon et de comprendre son lien avec les bâtiments monastiques.

Imitant peut-être en cela le duc de Bourbonnais Louis II et son frère, le «bâtard » de Bourbon ${ }^{9}$, les grandes familles locales multiplient les fondations de chapellenies à partir du dernier quart du XIV ${ }^{e}$ siècle dans les deux collatéraux sud de la nef. Les chapelles gothiques examinées en 2007 étaient séparées par un mur bahut supportant une grille, qui reliait la $2^{\mathrm{e}}$ pile de la colonnade externe sud à la colonne engagée sur dosseret lui faisant face sur le gouttereau ; la chapelle ouest ( $2^{\mathrm{e}}$ travée) était isolée de la même manière de celle qui occupait la travée d'angle sud-ouest, par une maçonnerie dont les fondations reposaient sur un des fours à chaux signalés plus haut. Cette chapelle ( $2^{e}$ travée) de la famille de Breuil/Chéry est d'abord placée sous le vocable de la Sainte-Trinité. Il s'agit du transfert en 1457 depuis la galilée occidentale, alors démolie, de l'ancienne chapelle de Breuil et des défunts qu'elle contenait, par Jean et sa nièce Suzanne de Breuil, épouse de Jean Foulé, maître d'hôtel du prieur commendataire de Souvigny, le cardinal archevêque de Lyon Charles de Bourbon ${ }^{10}$, troisième fils du duc Charles $\mathrm{I}^{\mathrm{er}}$. Après extinction du lignage, en 1511, le chapitre conventuel autorise Gilbert de Chéry à occuper cet emplacement avec sa famille et à le dédier à saint Antoine. Même si un drain du XIXe siècle a emporté un tiers du sous-sol, on voit que cette chapelle de famille est densément occupée par trois à quatre nappes de sépultures gothiques et modernes, où l'on observe des hommes, des femmes et des enfants même en bas âge. Dans la $3^{e}$ travée ouvrant sur la galerie orientale du cloître du XV ${ }^{e}$ siècle, les inhumations y sont moins nombreuses; on citera néanmoins un défunt enterré avec 
une bulle en plomb de Clément VII (1378-1394) ${ }^{11}$ et un ecclésiastique de l'époque moderne, dont le cercueil est orienté est-ouest et qui porte des bottines. Les deux sépultures les plus anciennes sont alignées au pied du gouttereau sud du $\mathrm{XI}^{\mathrm{e}}$ siècle, notamment une cuve monoxyle remployée sans couvercle.

Les quelques vestiges dans le vaisseau central à l'ouest du tombeau des saints abbés Le décapage du vaisseau médian de la nef a révélé la présence de multiples trous de poteaux des échafaudages et des fosses correspondant aux différentes campagnes de construction et de voûtement de l'édifice. Située dans l'axe, dans la $4^{e}$ travée, une empreinte rectangulaire pourrait correspondre au pied unique d'un autel de la SainteCroix, qui aurait fonctionné aux XI $\mathrm{XI}^{\mathrm{e}} \mathrm{XII}{ }^{\mathrm{e}}$ siècles. Une seule sépulture privilégiée du bas Moyen Âge, celle d'une femme (donatrice laïque ?), est apparue dans l'axe du haut vaisseau dans la $5^{\mathrm{e}}$ travée, à l'ouest du tombeau des saints abbés. Les aménagements de régulation hydraulique, motivés par les importants ruissellements sur le substrat argileux, ont déjà été signalés dans la campagne 2006. Nous avons mis au jour en 2007 le reste du drain du XVI ${ }^{e}$ siècle, qui collecte les eaux de pluie au nord du gouttereau septentrional du XII ${ }^{e}$ siècle ; il traverse le vaisseau central et les collatéraux sud dans la $4^{e}$ travée ( $3^{e}$ du bas-côté externe) et récupère l'exutoire (XII ${ }^{e}$ siècle) déjà mentionné, qui traverse les fondations du gouttereau sud pour évacuer les eaux dans le cloître. Les restaurateurs du milieu du $\mathrm{XIX}^{\mathrm{e}}$ siècle remploient à leur tour cette sortie pour aménager un grand drain coffré de dalles collectant les eaux du parvis occidental; la large tranchée de ce dernier, traversant obliquement les travées fouillées en profondeur du collatéral externe, bouleverse la stratigraphie et les tombes jusqu'au substrat.

L'ensemble des données collectées est en cours d'étude et nous prévoyons une exposition en 2009 au musée des Pays de Souvigny, après l'achèvement de l'examen, en juin 2008, du caveau du bâtard de Bourbon (Jean de Rochefort, $†$ 1375) et de son contenu ${ }^{12}$.

\section{NOTES}

1. Cf. P. CHEVAlieR, M. ČAUŠEVIĆ-BUlly, M. DUPUis, L. FIOCCHI et O. LAPIE, « Priorale SaintPierre de Souvigny (Allier). Étude archéologique de la nef, première tranche », Bulletin du Centre d'études médiévales d'Auxerre, 11 (2007), p. 71-77.

2. La campagne 2007 a employé quatre archéologues et un anthropologue pendant les quatre mois de terrain, la gestion passant par l'UMR 5594-ARTeHIS du CNRS ;

P. Chevalier a assuré la direction du chantier, dont la logistique a été confiée à

l'Association AMBRES. Nous remercions Amélie Berthon, Arlette Maquet, les étudiants et les bénévoles venus de France, de Chine et du Québec, mais aussi les collègues - au total 53 personnes -, qui nous ont offert leur temps et leurs efforts.

3. P. CHEVAlier et al., " Priorale Saint-Pierre de Souvigny... », op. cit., p. 73.

4. L'analyse a été réalisée à Zagreb par l'Institut Rudjer Bošković. 
5. Voir, en dernier lieu : P. CHEVALIER, « Les tombeaux et les monuments funéraires médiévaux des saints abbés Mayeul et Odilon de Cluny ", Hortus artium medievalium, 10 (2004), p. 119-132.

6. Les sondages réalisés dans le cloître par Sophie Hettiger (AFAN), en 1992, ont révélé la présence dans cet alignement d'un drain en coffrage de briques et tuiles plates à rebord, cf. S. HETTIGER, « Souvigny (Allier). Prieuré Saint-Pierre (fouilles 1992) », Archéologie médiévale, 24 (1994), p. 467-468.

7. Emmanuelle Boissart-Stankov, qui avait déjà examiné les enduits peints mis au jour autour du tombeau des saints abbés en 2002, achève cette étude ; cf. E. BOISSART-STANKOV, «Études archéologiques d'enduits peints : les exemples des chantiers de Cergy, Souvigny et Soissons ", Bulletin du Centre d'études médiévales d'Auxerre, 8 (2004), p. 40-41.

8. Voir N. STRATFORD, La frise monumentale romane de Souvigny, Souvigny, 2002.

9. P. CHEVAliER et al., « Priorale Saint-Pierre de Souvigny... ", op. cit., p. 76-77.

10. M. FAZY, Le livre des anniversaires du prieuré de Souvigny, Moulins, 1937, p. 29-30.

11. Aucun document écrit n'atteste cette pratique rare, toujours antérieure au milieu $\mathrm{du} \mathrm{XV}^{\mathrm{e}}$ siècle et qui accompagne les poussées les plus sévères de la Peste noire. Elle semble être liée à un type particulier d'indulgence accordé pour des œuvres charitables et de prière pour les morts, à partir de la fin du XIII ${ }^{\mathrm{e}}$ siècle et pendant tout le $\mathrm{XIV}^{\mathrm{e}}$ siècle, cf. R. GILCHRIST et B. SLOANE, Requiem. The medieval monastic cemetery in Britain, Londres, 2005, p. 94-96.

12. Cette opération courte sera menée conjointement par Audrey Baradat, Patrice George (Inrap) et Philippe Charlier (médecin légiste).

INDEX

Index géographique : France/Souvigny

Mots-clés : nef, priorale, Saint-Pierre de Souvigny 